\title{
A patient with difficulty of object recognition: semantic amnesia for manipulable objects
}

\author{
A. Yamadori, Y. Yoneda, H. Yamashita ${ }^{1}$ and K. Sugiura ${ }^{1}$ \\ Neurology Service and Neuropsychology Section', Hyogo Brain and Heart Center at Himeji, \\ Japan
}

Correspondence to: A. Yamadori, Neurology Service, Hyogo Brain and Heart Center at Himeji, 520, Saisho-Ko, Himeji, Hyogo-ken, 670, Japan

\begin{abstract}
We studied a patient who had recognition difficulty for manipulable objects. MRI showed a lesion in the left occipito-parietotemporal area. Differential diagnosis of agnosia, aphasia and apraxia is discussed. We believe this "object meaning amnesia" constitutes a distinct subtype of semantic amnesia.
\end{abstract}

Keywords: Multi-modal agnosia - Object meaning amnesia - Object recognition - Semantic memory

\section{INTRODUCTION}

Difficulty of object recognition can occur as a feature of several clinical syndromes. Agnosia is a disturbance of object identification limited to a single sensory modality. Thus a visual agnosic should be able to identify a visually unidentifiable object through tactile manipulation. Aphasia is defined as a difficulty of language manipulation. Thus an anomic aphasic may not be able to name a stimulus object regardless of the sensory modality through which it is presented. However, he knows what it is and should be able to demonstrate this knowledge by paraphrasing, gesture, or actual use. In ideational apraxia a patient may show difficulties in manipulating an object but knows what he is manipulating. Recently we have encountered a patient who could not name objects in any sensory modality, who could not describe by language or gesture what he was dealing with, and who could not use or classify the objects into an appropriate category.

\section{CASE PRESENTATION}

A 75-year-old right handed retired civil servant (M.Y.) suddenly developed difficulty in finding words on 1 February 1990. A CT scan of the brain taken next day revealed a left occipital low density area indicating infarction. He was referred to our hospital on 14 February for further evaluation and treatment.

On admission M.Y. showed a complete right homonymous hemianopsia. He was awake and attentive but was slow in response. He was oriented to time. He knew which city he was in but did not know the name of the hospital. His digit span was five forwards. He could remember none of the three words he had been asked to remember $5 \mathrm{~min}$ before. His spontaneous speech was well formulated without dysarthria or grammatical abnormalities. His comprehension of verbal questions and commands was fair. In naming he was correct in only three objects out of 10 . Repetition of word and sentence was good. He showed severe difficulty in writing and reading. Reading did not improve by kinesthetic facilitation. He could copy a cube figure correctly. He showed no buccofacial or ideomotor apraxia. Details of the neuropsychological data accumulated during the second and third month when his condition had stabilized are summarized in Table I.

The most striking and puzzling feature of M.Y.'s behaviour was his peculiar attitude towards some of the household utensils presented to him for naming. He would pick them up and gaze at them for a prolonged time and finally complain he didn't know what they were. Or he would produce rather wild paraphasias. For instance he described a key as something to measure time. He was unable to demonstrate how it was used and seemed totally at a loss as to what its true nature was. We conducted a small study on this aspect.

\section{Identification of manipulable objects}

On the seventh hospital day we tested M.Y.'s object identification ability for six common manipulable objects, i.e. a bundle of keys, a pair of scissors, a wrist watch, a Japanese type abacus, a box of matches and a small transistor radio. For each object visual naming, verbal description of its nature, demonstration of its use by gesture, tactile naming (except for box of matches and radio), dem- 
TABLE I. Result of neuropsychological tests (1990.3-1990.4)

\begin{tabular}{llc}
\hline Verbal memory & & \\
Digit span & Forward & 5 \\
Recent memory & 3 names after 5 min & 0 \\
Miyake Retention Test & Related 10 pair words & $1 / 4 / 2$ \\
& Unrelated 10 pair words & $0 / 0 / 0$ \\
Visual memory & & \\
BVRT & Copy & 1010 \\
& Immediate recall & $2 / 10$ \\
Language & & \\
Visual naming & Pictures & $55 / 90$ \\
Tactile naming & & $9 / 20$ \\
Oral reading & Kana letter & $17 / 46$ \\
& Kanji letter & $3 / 20$ \\
Intelligence & & \\
MMST & & $17 / 30$ \\
WAIS & Performance & 77 \\
& Verbal & 66 \\
RCPM & Total & 68 \\
& & $24 / 36$ \\
\hline
\end{tabular}

Miyake Retention Test presents 10 paired words and has the patient recall the other from a stimulus word. Three trials were performed successively. BVRT, Benton Visual Retention Test; MMST, Mini-Mental State Test of Folstein; WAIS, Wechsler Adult Intelligence Scale; RCPM, Raven Colored Progressive Matrices.

TABLE II. Result of object identification test

\begin{tabular}{lcccccc}
\hline & $\begin{array}{c}\text { Visual } \\
\text { naming }\end{array}$ & Describe $^{1}$ & $\begin{array}{c}\text { Tactile } \\
\text { naming }\end{array}$ & $\begin{array}{c}\text { Use } \\
\text { Auditory } \\
\text { naming }\end{array}$ & Point \\
\hline Key & - & - & - & - & - & - \\
Scissors & - & - & - & + & & - \\
Watch & - & - & - & - & - & + \\
Abacus & - & - & - & + & - & + \\
Matches & - & - & & + & - & + \\
Radio & - & - & & + & - & + \\
\hline
\end{tabular}

1 This item covers verbal description and gestural demonstration tasks. M.Y. did not succeed either way.

onstration of its actual use, auditory naming (except scissors) and matching its verbal name with an appropriate object (pointing) were tested in that order.

As is shown in Table II he failed to identify the bundle of keys by any means. For the scissors he was able to demonstrate their use correctly but could not explain what they were or what they were used for. For the other four items he could either use them or point to them correctly. Without exception, however, his response was hesitant and he kept complaining he did not know them for sure even when his use of them or pointing was correct. Finally he was asked to make sketches of each object. His drawings were all appropriate revealing his normal visual and constructive ability (see Fig. 1). However, this task did not lead him to better comprehension of the stimuli.

On the 34th day post onset we tested his ability of cate-
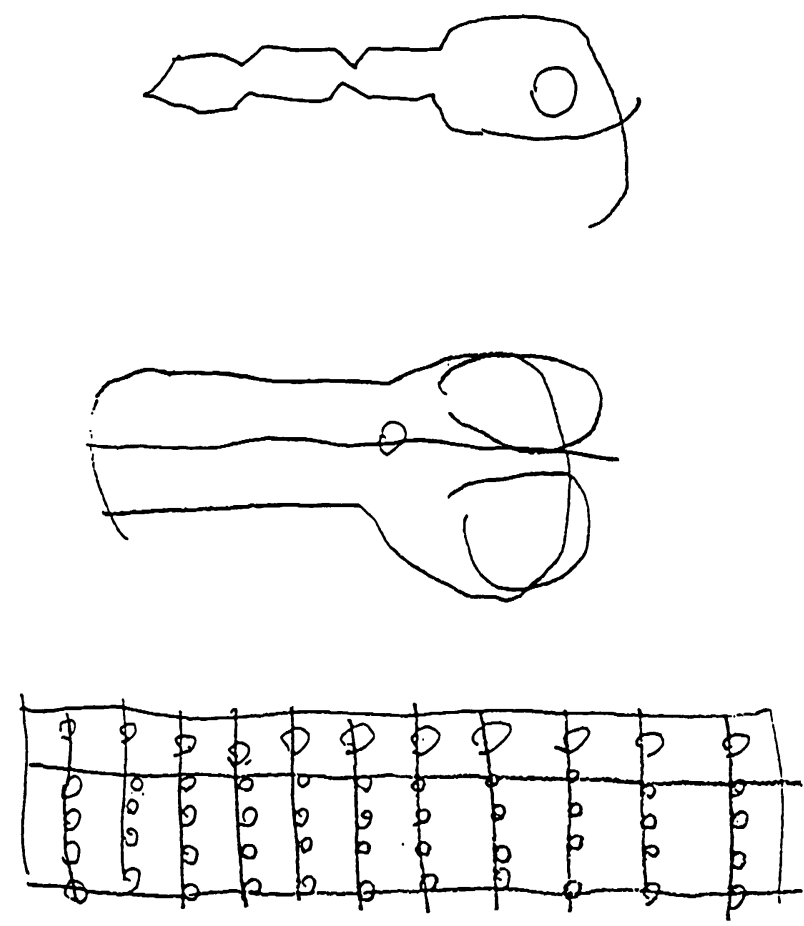

FIG. 1. Examples of his drawings of unidentifiable objects. From top to bottom: key, scissors, and Japanese style abacus.

gorization. We used 10 objects that could be classified into five pairs (a small radio, a cassette tape, a seal, a case of seal ink, a tea cup, a teapot, a knife, a fork, a can, and a can opener). All items were randomly laid out in front of him. He was asked to make a pair as he considered appropriate. No time limit was imposed. He was free to manipulate all of them. He assembled only one pair of them correctly, i.e. knife and fork. For the rest he assembled tea cup and seal ink, tea pot and can, cassette tape and can opener, seal and radio together with extreme reluctance and confessed it was very difficult. Naming was not tested in case paraphasia should affect his performance. On another occasion, he was asked to classify another 10 items (pencil, eraser, tooth brush, tooth paste, wallet, one thousand yen note, hammer, nail, box of matches and candle) into five groups. He assembled only two pairs correctly (wallet-thousand yen; nail-hammer). Naming was correct for two items, i.e. thousand-yen note and matches, and demonstration of use was correct for three, i.e. pencil, hammer and tooth paste. The correct use, however, did not necessarily mean correct recognition. For instance he demonstrated the use of the pencil correctly, but did it in a hesitant way and finally matched it with the tooth paste.

\section{Visual and tactile naming}

To probe the possibility of category selectivity, we tested his naming ability for 90 pictures of objects consisting of nine categories with 10 items for each category. The nine 
TABLE III. Result of naming pictures by category

\begin{tabular}{lcc}
\hline Categories & No of cards & Correct \\
\hline Color & 10 & 4 \\
Clothes & 10 & 7 \\
Food & 10 & 7 \\
Kitchen & 10 & 8 \\
Object & 10 & 7 \\
Body part & 10 & 5 \\
Animal & 10 & 7 \\
Vehicle & 10 & 6 \\
Plant & 10 & 4 \\
Total & 90 & 55 \\
\hline
\end{tabular}

categories consisted of color, clothing, food, kitchen utensils, manipulable objects, body parts, animals, vehicles and plants. His naming was poor in all categories (see Table III).

Also tactile naming was tested for 20 objects. Six were correctly named. Three others were correctly described.

\section{Neuroimaging study}

A CT scan and a MRI study demonstrated a large continuous lesion in the left occipital lobe, left posteroinferior parietal lobe and portion of the left medial temporal lobe affecting the hippocampus. In addition MRI revealed a couple of small lesions in the left frontal subcortical area. A ${ }^{123}$ I-iodoamphetamine SPECT (single photon emission computed tomography) study of the brain revealed low uptake in the left occipito-temporo-parietal area. Arteriography demonstrated occlusion of the left posterior cerebral artery at its origin.

\section{Clinical course}

M.Y.'s difficulty in recognizing manipulable objects improved slowly. He was discharged on 21 May. On 9 August 1990, six months post onset, he was seen in the outpatient clinic by one of us (A.Y.). When he was shown a Japanese style writing brush for identification, he looked at it for quite a long time and finally said he didn't know what it was. When he was asked to demonstrate how it should be used, he held it correctly but could not mime writing. When pressed for use, he started brushing the face of his wrist watch on his left hand saying that he was not sure that was correct. Becoming impatient his wife supplied the object name but he did not seem to comprehend. According to her he used to use a writing brush quite often. One year post onset we tested his object recognition capacity with the same method as shown in Table II employing 10 manipulable objects. He was unable to identify two items (table tennis ball and key) by any means.

\section{DISCUSSION}

These observations demonstrate M.Y.'s marked difficulty

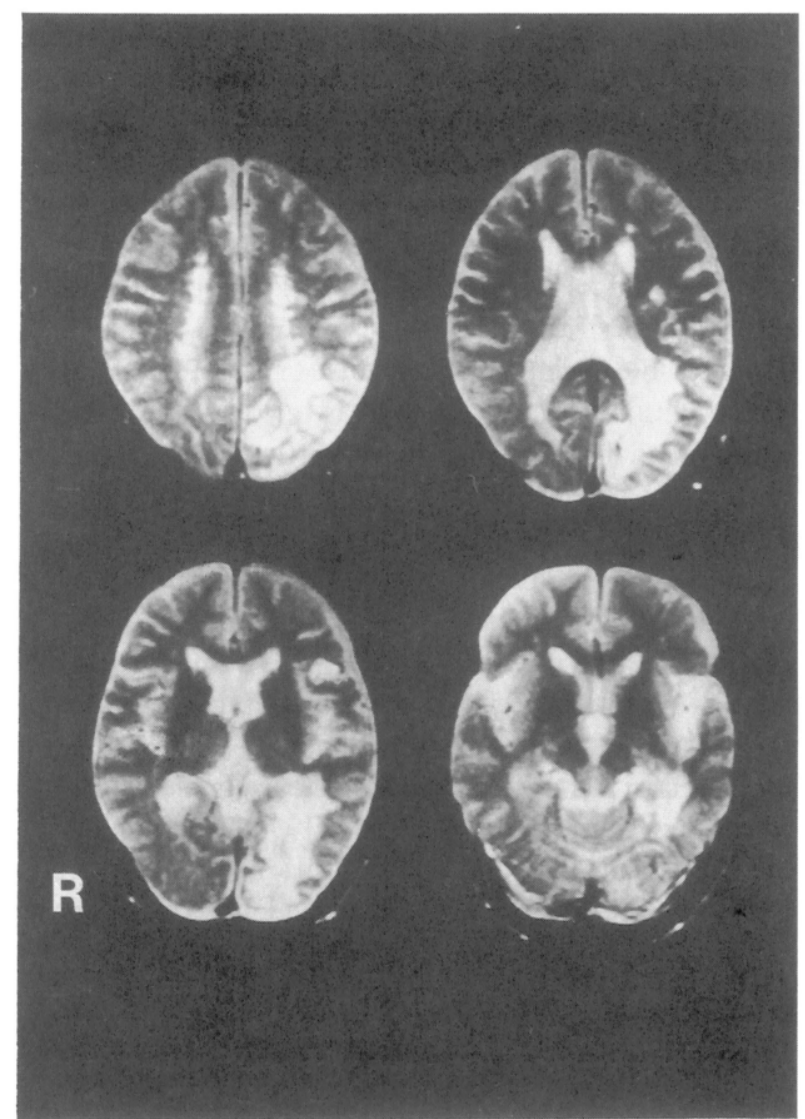

FIG. 2. 1.0 Tesra MRI image with T2 emphasis demonstrates high signal area in the left occipital lobe, extending upward into the parietal lobe and forward into the medial temporal lobe. The right side of the images corresponds to the left side of the brain.

in identifying familiar manipulable objects. The difficulty did not improve by increasing sensory information and verbal cues. As far as his behavioral reaction to a given object was concerned, he gave us the impression that he had no mental image (or concept) of an object to which he could refer a stimulus with confidence.

Differential diagnosis includes associative visual agnosia, multi-modal agnosia, two-way anomia, and ideational apraxia. M.Y. could not recognize a stimulus neither via vision nor other sensory modalities, ruling out the diagnosis of associative visual agnosia. Since he could not identify objects through any sensory modalities, multimodal agnosia could be a good candidate (Feinberg et al., 1986; Sirigu et al., 1991). However, the definition of agnosia presupposes a preserved concept of object. In a detailed study of a similar case Sirigu and her colleagues concluded that their patient had relatively intact representations of objects judging from the data of verbal tasks, thereby placing their case in the category of multi-modal agnosia (Sirigu et al., 1991). M.Y.'s difficulty did not improve by any means making it difficult to assume that his concepts of objects were intact. Two-way anomia is a 
difficulty in evoking a name from a stimulus and matching a heard name to a stimulus, which occasionally mimics agnosia. The concept of two-way anomia was first proposed to explain modality specific naming difficulty seen in certain types of disconnection syndromes (Geschwind, 1967) and later expanded to supra-modal anomia coupled with word meaning impairment (Yamadori and Albert, 1973). This is strictly an aphasic disturbance and patients should be able to demonstrate the use of a stimulus or show its recognition by gesture, which is beyond M.Y.'s capacity. Ideational apraxics may show difficulty in manipulating a single object but they should know what they are manipulating (De Renzi and Lucchelli, 1988).

M.Y. is unique in that he had unequivocal difficulty in comprehending the meaning of manipulable common objects. It is true that he did sometimes show the correct usage of an object. Or he would occasionally point to a correct item. He remained puzzled, however, even when he pointed to a correct object for a heard name. That this type of partial hit does not necessarily mean true comprehension is beautifully described by Sirigu et al. (1991). His lack of comprehension was further evidenced by his gross errors in categorizing manipulable objects. In our experience even a severe Wernicke's aphasic would not fail in this type of task. We assume that M.Y. either could not recall the concept of a stimulus or could evoke only a fragment of its various attributes. For its full recognition a systematic and stable association of modal percepts and lexicon must be a prerequisite.

This impairment is akin to the so called semantic amnesia proposed by Warrington (1975). It is defined as disturbance at the level of knowledge or concept. Patients with this syndrome show difficulty in comprehending the nature of an item presented to them. Principal methods used to prove this type of semantic impairment is to test naming, pointing or verbal description capacity using numerous test stimuli, in which a test performed has been limited to one modality at a time (Warrington and Shallice, 1984; Warrington and McCarthy, 1987; De Renzi et al., 1987; Grossi et al., 1988). The resulting data have been discussed in terms of modality specificity like semantic amnesia limited to visual modality (Warrington, 1975) or category specificity like selective disturbance of knowledge about living things and foods (Warrington and Shallice, 1984). Unfortunately these studies lack detailed behavioral accounts about how these patients responded to a particular manipulable object under such conditions that every sensory modality and verbal cue is freely available.

To our knowledge in depth symptomatological analysis of this type of identification difficulty for manipulable objects has rarely been reported. Demented patients in advanced stage may sometimes show similar difficulties (Hamanaka, 1986). We once had a chance to observe a similar symptom in a post traumatic patient (Yamadori,
1988). We could not make the patient understand a key either through any sensory modalities or through language. We believe this aspect of cognitive disturbance should be treated separately from the general concept of semantic amnesia in its current usage. Here our concern is limited to a problem of the identification of three dimensional real objects and necessarily directed to impairment of cross-modal or supra-modal dimension. We propose to call this symptom object meaning amnesia. Degradation of a system to integrate constituent modal attributes of an object into a single mental representation must be a cause of this peculiar syndrome.

M.Y.'s brain lesion revealed by the MRI study was strictly unilateral occupying a relatively wide area from the parieto-occipital lobe to the posteromedial portion of the temporal lobe of the left hemisphere. It destroyed the left visual cortex totally and the left hippocampal region partially. It was in a strategic position to disconnect the more superior Wernicke's area, the auditory association area, the tactile association area and the right hemispheric visual association area. The resulting functional dissociation among the memory, speech, tactile, auditory and visual systems may explain this object meaning amnesia. Most of the previous reports of so called semantic amnesia either had bilateral lesions in the temporal lobes (De Renzi et al., 1987; Hamanaka, 1986; Pietrini et al., 1988) or left unilateral parietal lesion (Grossi et al., 1988). This may be the first report of semantic amnesia for manipulable objects with a strictly unilateral lesion.

\section{Acknowledgement}

This research was supported by funds from the Ministry of Education, Japan.

\section{REFERENCES}

De Renzi E and Lucchelli F (1988) Ideational apraxia. Brain, 111, $1173-1185$.

De Renzi E, Liotti M and Nichelli P (1987) Semantic amnesia with preservation of autobiographic memory. A case study. Cortex, 23, 575-597.

Feinberg TE, Rothi LJG and Heilman KM (1986) Multimodal agnosia after unilateral left hemisphere lesion. Neurology, 36, 864-867.

Geschwind N (1967) The varieties of naming errors. Cortex, 3, 97-112.

Grossi D, Trojano L, et al. (1988) Selective "semantic amnesia" after closed-head injury. A case report. Cortex, 24, 457-464.

Hamanaka T (1986) Clinical Neuropsychiatry. Igaku-Shoin, Tokyo (in Japanese).

Pietrini V, Nertempi P, et al. (1988) Recovery from herpes simplex encephalitis: selective impairment of specific semantic categories with neuroradiological correlation. Journal of Neurology, Neurosurgery and Psychiatry, 51, 1284-1293.

Sirigu A, Duhamel J-R and Poncet M (1991) The role of sensorimotor experience in object recognition. A case of multimodal agnosia. Brain, 114, 2555-2573. 
Warrington EK (1975) The selective impairment of semantic memory. Quarterly Journal of Experimental Psychology, 27, 635-657.

Warrington EK and Shallice T (1984) Category specific semantic impairments. Brain, 107, 829-854.

Warrington EK and McCarthy RA (1987) Categories of knowl- edge. Further fractionations and an attempted integration. Brain, 110, 1273-1296.

Yamadori A (1988) Selective impairment of semantic memory. Clinical Psychiatry, 17, 1299-1305 (in Japanese).

Yamadori A and Albert ML (1973) Word category aphasia. Cortex, 9, 112-125. 


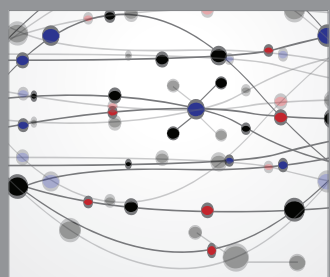

The Scientific World Journal
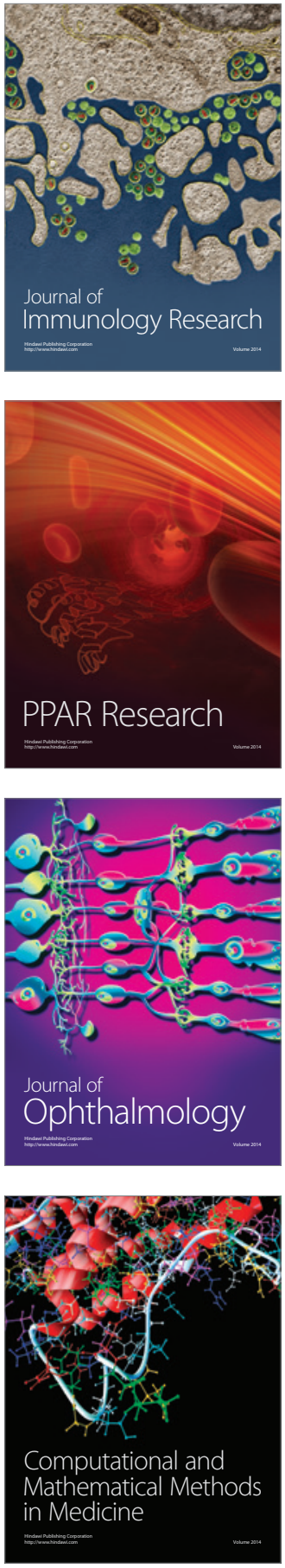

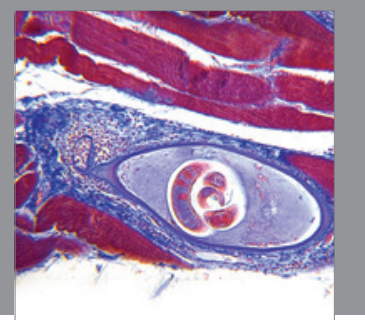

Gastroenterology

Research and Practice
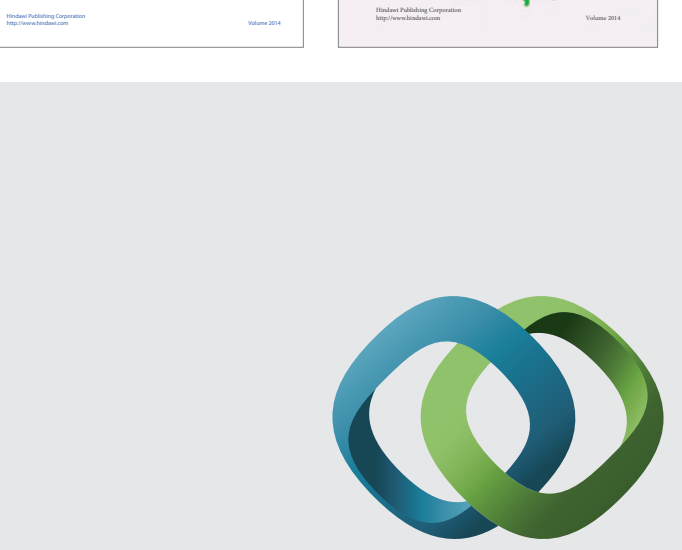

\section{Hindawi}

Submit your manuscripts at

http://www.hindawi.com
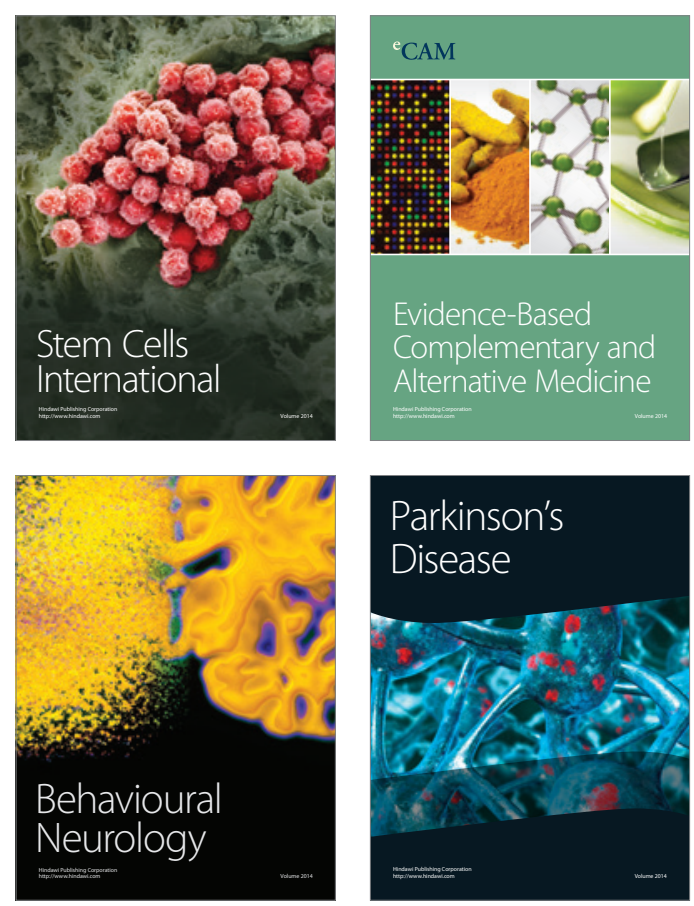

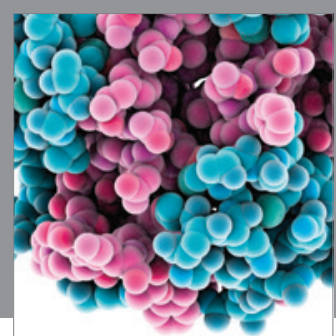

Journal of
Diabetes Research

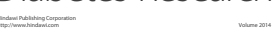

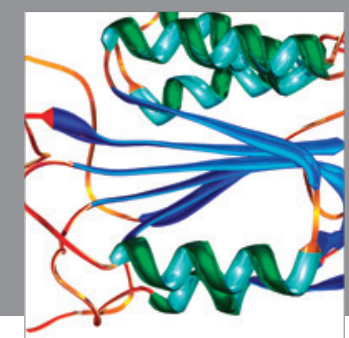

Disease Markers
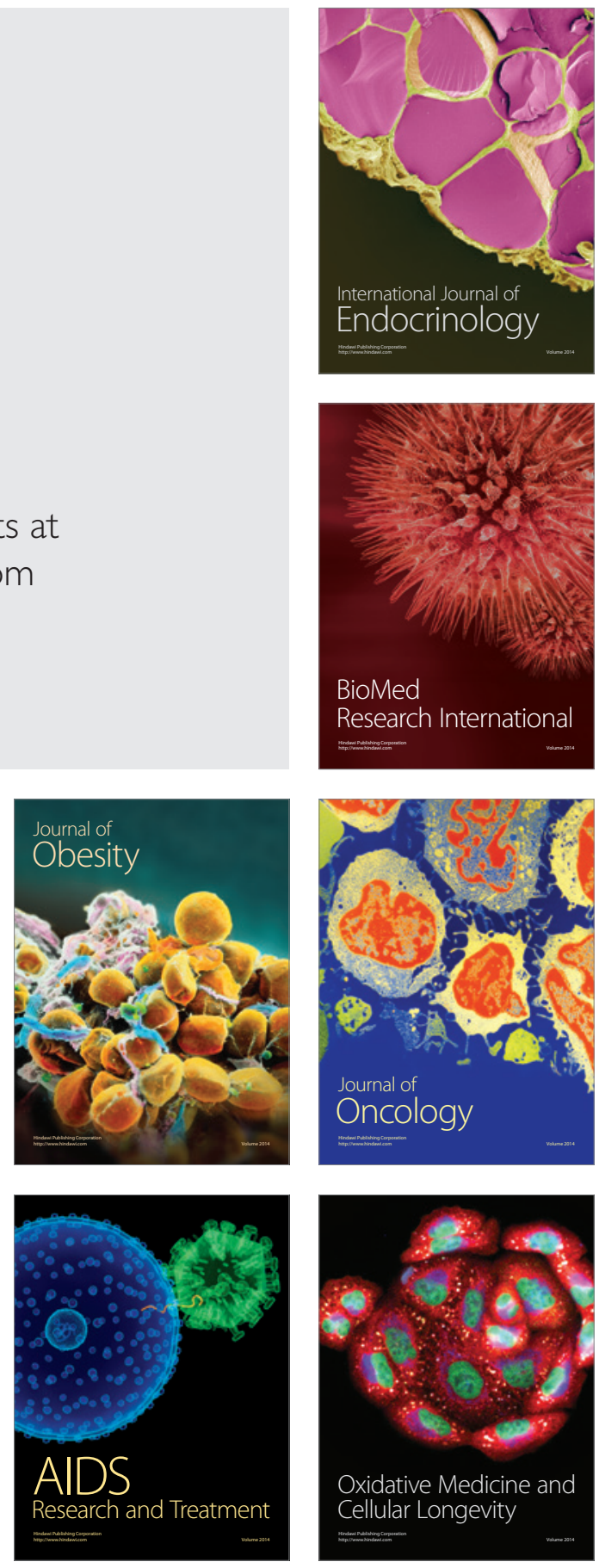